\title{
MANEJO ODONTOLÓGICO NA TERAPIA DO BRUXISMO
}

\author{
Deisiany Rupolo da Cunha ${ }^{1}$ \\ Germana Vieira Sousa ${ }^{2}$
}

RESUMO: O bruxismo é caracterizado pelo movimento involuntária de apertar ou ranger os dentes, sendo classificado em bruxismo do sono (ranger) e bruxismo diurno (apertar), este último sendo frequente em momentos de tensão ou estresse. Os episódios do bruxismo podem acarretar danos na articulação temporomandibular, desgastes dentários entre outros. No entanto, essas sequelas podem ser tratadas e até evitadas quando o diagnóstico é feito na fase inicial. Não existe um protocolo único para o tratamento do bruxismo; desta forma, os diferentes meios terapêuticos, devem ser diferenciadas para cada paciente. Por meio de diagnóstico do paciente, pode-se direcionar o manejo mais eficaz, no entanto, mais pesquisas devem ser realizadas para comprovação da eficácia dos mesmos. Este trabalho compõe-se de um estudo de revisão de literatura com consulta aos bancos de dados online do qual, foram incluídos 31 artigos com publicações de 1990 a 2021 com o objetivo de apresentar as principais formas terapêuticas de manejo odontológico do bruxismo.

Palavras Chave: Bruxismo. Manejo da Dor. Terapêutica.

ABSTRACT: Bruxism is characterized by the involuntary frequency of clenching or grinding the teeth, being classified into sleep bruxism (grinding) and daytime bruxism (grinding), the latter being frequent in moments of tension or stress. The episodes of bruxism cause damage to the Temporomandibular Joint, dental wear, among others. However, these sequelae can be treated and even avoided when diagnosed on the initial face. There is no single protocol for the treatment of bruxism; in this way, the different therapeutic means must be differentiated for each patient. Through patient diagnosis, one can direct the most effective management, however, more research must be carried out to prove their effectiveness. This work consists of a literature review study that consulted online databases, which included 31 articles published from 1990 to 2021 with the aim of presenting the main therapeutic forms of dental management of bruxism.

Key Words: Bruxism. Pain management. Therapeutic.

\begin{tabular}{l}
\hline Graduando em Odontologia, Unifasipe Centro Universitário, Mato Grosso, \\
deisilider@hotmail.com. \\
2 Professora orientadora. Mestre em DTM e Dor Orofacial, Professora do Estágio em \\
Odontopediatria da UNIFASIPE Centro Universitário, Mato Grosso. E-mail: \\
germanavs@yahoo.com.br
\end{tabular}




\section{INTRODUÇÃO}

O bruxismo é uma desordem da musculatura da mastigação, fazendo com que o paciente tenha apertamento dental ou ranger dos dentes. É mais comum durante o sono, porém, também pode acontecer durante a vigília, podendo provocar sequelas no sistema estomatognático, desgastes dentais, periodontopatias, e dores articulares e fracassos clínicos (GAIDA, PS. Bruxismo et al., 2004).

O termo bruxismo origina-se do grego brychein, que significa ranger de dentes, porém, outros nomes têm sido usados para descrever este quadro: neurose do hábito oclusal, neuralgia traumática, bruxomania, friccionar-ranger de dentes, briquismo, apertamento e parafunção oral (GAIDA, PS. Bruxismo et al., 2004).

O bruxismo do sono se diferencia do bruxismo diurno por envolver distintos estados de consciência, sono e vigília; e estados fisiológicos com diferentes influências na excitabilidade oral motora. Assim o bruxismo do sono é uma atividade inconsciente de ranger ou apertar os dentes, com produção de sons, enquanto o indivíduo encontra-se dormindo. Já o bruxismo diurno é caracterizado por uma atividade semivoluntária da mandíbula, de apertar os dentes enquanto o indivíduo se encontra acordado, em que geralmente não ocorre o ranger de dentes, e está relacionado a um tique ou hábito (DORLAND, WA. et al., 200o, BLINI CC. et al., 2010).

Pode ser tratado por diferentes linhas de tratamentos, como: tratamento farmacológico, psicológico e odontológico. Tratamento farmacológico é utilizado para casos agudos e graves e consiste no uso dos fármacos por um período curto de tempo. Benzodiazepínicos, anticonvulsivantes, betabloqueadores, drogas dopaminérgicas, antidepressivos e relaxantes musculares são mais comumente usados. A psicoterapia é um tratamento baseado em fortes evidências e inclui a terapia comportamental baseada na higiene do sono, o controle de estresse e técnicas de relaxamento, o tratamento é baseado em fortes evidências. Os tratamentos odontológicos para bruxismo incluem ajuste de oclusal, restauração da superfície do dente, tratamento ortodôntico e placa oclusal (CHASE MH, MORALES et al., 2000). 
Esse estudo tem por objetivo identificar as principais formas terapêuticas no manejo do bruxismo, demostrando assim, quais meios terapêuticos o profissional odontologistas deve indicar conforme a real necessidade do paciente. Onde através de uma revisão integrativa da literatura, com a intenção de aprofundar o conhecimento referente ao manejo dos pacientes com o Bruxismo em que foi utilizado por meio de pesquisas como revistas e artigos, usando como plataforma Google acadêmico, Scielo. Como critério de inclusão para a escolha dos materiais utilizados, foram realizadas pesquisas online utilizando palavras chaves como: Bruxismo, Tratamento do bruxismo, Formas Terapêuticas, Como critério de exclusão, não foram utilizados artigos publicados há mais de 30 anos.

\section{REVISÃO DE LITERATURA}

\subsection{Conceito do Bruxismo}

O bruxismo é definido como uma atividade repetitiva dos músculos mastigatórios caracterizada pelo ranger e/ou apertar de dentes com diferentes manifestações. $\mathrm{O}$ comportamento mostrou associação com diversos distúrbios caracterizando sua complexa fisiopatologia. O apertamento dos dentes é um fechamento forte das dentições opostas em relação estática da mandíbula à maxila, em intercuspidação máxima ou uma posição cêntrica, enquanto a moagem da dentição é um forte fechamento oposto em um relacionamento maxilo-mandibular dinâmico, em que o arco mandibular se move através de várias posições excursivas. Todas as formas de bruxismo implicam vigorosamente entre as superfícies de mastigação dos dentes superiores e inferiores (LAVIGNE GJ. Et al., 2008).

Ambas as manifestações circadianas do bruxismo refletem características psicológicas específicas como ansiedade, sensibilidade ao estresse e padrões excitatório. A avaliação e diagnóstico do bruxismo é muitas vezes um verdadeiro desafio. Em geral, a avaliação é baseada em relatos de sons de trituração dos dentes durante o sono e a presença de sinais e sintomas clínicos. O bruxismo noturno pode estar relacionado a síndrome da apnéia obstrutiva do sono, síndrome das pernas inquietas e refluxo gastroesofágico. Assim como, a ingestão de álcool, uso de tabaco, 
cafeína e alguns antidepressivos também tem um impacto no desenvolvimento do bruxismo. Inicialmente, o método de abordagem genética do bruxismo era um estudo da hereditariedade comportamental, mostrando que a condição era compartilhada por membros imediatos da mesma família. Neste contexto, considerando que o bruxismo também é concomitante com outros distúrbios (LOPES, NM de A et al., 2018).

Os efeitos prejudiciais oriundos desse comportamento mostram-se como justificativa para condutas de controle. Desgastes nos dentes, fraturas de reabilitações protéticas, aumento de dores orofaciais, sensibilidade dentária, cefaleia tensional temporal e ruídos sonoros durante o sono são consequências observadas em bruxômanos (LOBBEZOO F, LAVIGNE GJ et al., 1997).

\subsection{Evidências Genéticas}

Estudos revelaram que $20 \%$ a $50 \%$ das pessoas diagnosticadas apresentaram pelo menos um membro imediato de sua família com a mesma parafunção. A maioria dos pacientes, em conversa com os seus dentistas sobre o seu problema/ hábito de ranger dos dentes, diz que um ou mais membros da sua família também range os dentes. Por norma, esta informação é dada de forma espontânea, sem qualquer questão sobre a mesma por parte do paciente. A possibilidade de que a doença pode ser de família é claramente algo que as pessoas tendem a perguntar (LOBBEZOO F, LAVIGNE GJ et al., 1997).

A primeira prova genética do bruxismo procuraria elucidar a hereditariedade do comportamento. A influência genética no bruxismo também foi demonstrada por estudos de concordância e a presença da mesma característica fenotípica em pares de gêmeos geralmente monozezyóticos. A base para essa conclusão é que monozigóticos são geneticamente idênticos, enquanto dizigóticos compartilham somente 50\% do material genético. Assim como em estudos familiares, a maioria dos estudos de gêmeos concluíram que o bruxismo é parcialmente determinado por fatores genéticos MANFREDINI D. et al., 2013). 


\subsection{Fatores Psicológicos}

Estudos realizados com crianças bruxômanas, demonstraram que aproximadamente $82,76 \%$ apresentavam problemas de comportamento ou emocionais, necessitando assim de tratamento psiquiátrico e/ou psicológico. Pacientes com bruxismos, em sua maioria apresentam manifestações físicas e psicológicas de estresse, que se caracterizam por distúrbios de medo, de sono, mauhumor, preocupações, ranger de dentes e outros (LT, AGUIAR VNP, MACHADO FC, ROCHA CT, NEVES BG. 2018).

Quando o bruxismo é o resultado do fator central, um mapa biopsicossocial do paciente deve ser traçado, levando em consideração o comportamento diário e variações de humor, sendo estes fatores associados ao surgimento de episódios de bruxismo. Tem sido encontrado traços comuns como irritabilidade, pessimismo, medo, timidez, extravagancia e impulsividade. Sendo a boca um dos sistemas com potencial emocional refletindo, mesmo que inconscientemente, as emoções e frustrações do indivíduo. Nesse sentido, o bruxismo e outras funções irão se manifestar na forma de automutilação na resposta de fuga para expressar impulsos reprimidos (NASCIMENTO AM. et al., 2009).

\subsection{Formas de Manejo}

O profissional dentista, dispõe de alguns meios terapêuticos, para o manejo desta disfunção, sendo a mais comum as placas oclusais, no entanto outra alternativa tem apresentado efeito mais significativo, sendo alguns testes: acupuntura, medicação, técnicas fisioterápicas como relaxamento, Estimulação Elétrica Nervosa Transcutânea (GROSSMANN E. et al., 2012).

\subsection{Placas Oclusais}

O bruxismo está entre os principias fatores de dor não dentária na região orofacial, influenciando significativamente na qualidade de vida do paciente. $O$ uso de placas oclusais (Figura or,02) é o método mais usado no tratamento do bruxismo, atuando como forma de proteção dos dentes contra o desgaste patológico. Sua 
composição pode ser de material vinil ou acrílico rígido, se encaixando nos dentes inferiores e superiores, protegendo assim os dentes de atritos causados pelo bruxismo. No entanto o uso de placa oclusiva tem poder tratamento muito ameno, sendo praticamente paliativo, sendo apenas um minimizador dos efeitos colaterais, no tratamento da doença (FERREIRA JC. AFONSO. 2019)

Poucos estudos comprovam o uso dos dispositivos orais como forma eficaz no tratamento de pacientes bruxômanos, pois sua ação evita apenas o atrito dentário provocado pelos episódios de bruxismo, não tratamento o episódio em si, no entanto verifica-se a existência dos seus benefícios na prevenção ou retardamento dos desgastes dentários recorrentes dos episódios de bruxismo do sono (FERREIRA JC. AFONSO. 2019).

Figura or - Placa oclusiva, indicada para o bruxismo do tipo excêntrico (ranger dentário).

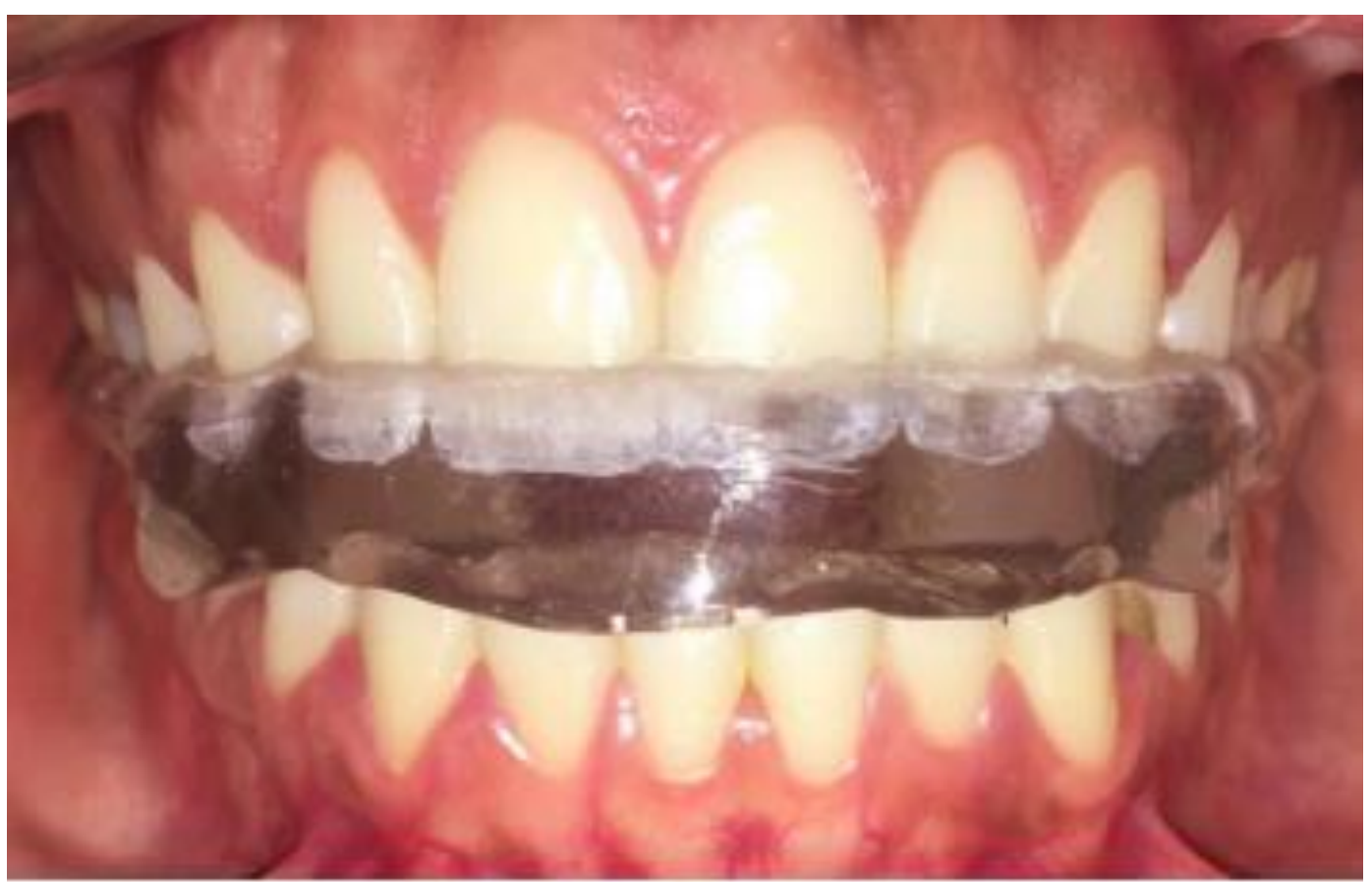

Fonte: VASCONCELOS; VENÂNCIO; SILVA, (2018) 
Figura 02 Placa para bruxismo, em silicone.

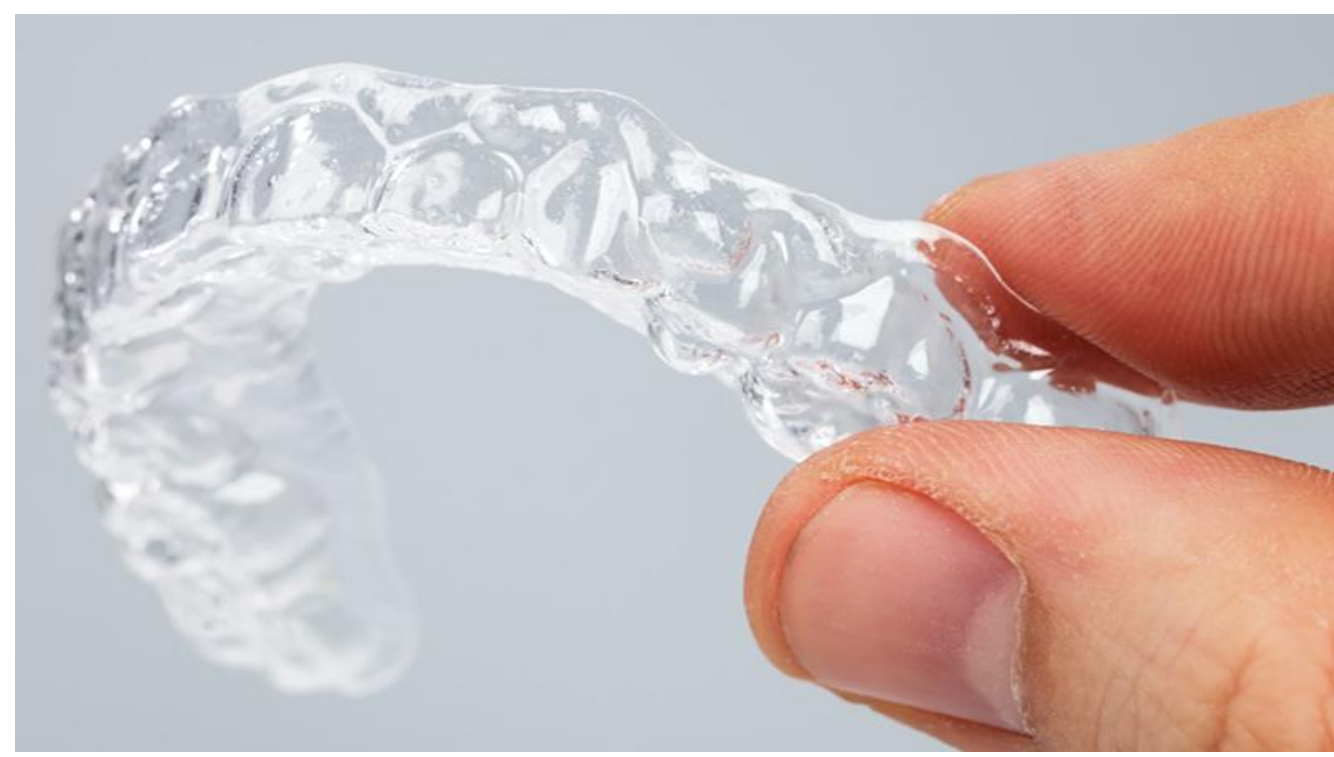

Fonte: LEITE (2019).

Existe atualmente no mercado, diversos modelos de placas oclusais para diminuir o ranger dos dentes nos pacientes bruxomanos. Sua confecção visa uma variação de encaixes, como no arco inferior e superior ou separadamente. Pode-se ainda, cobrir todos os dentes ou contar com cobertura parcial, visando assim desenvolver uma estabilidade das articulações temporomandibular, protegendo os dentes dos desgastes. Para se obter um resultado aceitável, o uso da placa dependerá de uma confecção precisa, uso correto pelo paciente, assim também como a escolha correta pelo profissional cirurgião dentista (LIMA, MCG. et al., 2020).

O uso da placa oclusiva, tem um tempo máximo de efeito, sendo nas duas primeiras semanas, após esse período tende - se a voltar ao ponto base no inicio do tratamento, com episódios de bruxismo. O uso prolongado da placa também pode acarretar outros sintomas, como dor, alteração da mordida e da oclusão. Seu uso é questionado por profissionais odontologistas, devido ao curto período de atuação e não tratamento da doença. Pois pode acarretar piora na respiração durante o sono, em pacientes acometidos com síndrome de apneia obstrutiva do sono, sendo a utilização de dispositivo de avanço mandibular uma alternativa para o tratamento de bruxismo a esse público (MACHADO E. et al., 20II). 
Apesar de que estudos comprovam o uso dos dispositivos orais como forma eficaz no de tratamento de pacientes bruxônomos, verifica-se a existência dos seus benefícios na prevenção ou retardamento do desgaste dentário recorrente dos episódios de bruxismo do sono (FERREIRA JC. AFONSO. 2019).

No entanto, a placa oclusal ainda é uma escolha viável, nos tratamentos iniciais de bruxismo, por não ser invasivo e pelo custo benefício que o tratamento oferece, sendo ainda um tratamento mais indicado, levando em consideração sua associação com outras terapias alternativas, apresentando um resultado satisfatório, reduzindo a intensidade e frequência da dor e melhorando a qualidade de vida do indivíduo que porta essa disfunção (SANTOS J, RECCO P, MOTA G, HOLANDA AV, JUNIOR VES. 2017).

\subsubsection{Acupuntura}

A acupuntura está inserida dentro da Medicina Tradicional Chinesa, sendo utilizada em diversos campos terapêuticos. Essa técnica não invasiva também tem apresentado eficácia no tratamento do bruxismo, agindo com a liberação de hormônios como, endorfina e serotonina, que atuam nos movimentos mandibulares, diminuindo o estresse dos músculos responsáveis pela mastigação. Para obter o efeito terapêutico, a técnica de acupuntura, através de pontos energéticos espalhados pelo corpo, com a utilização de sementes, agulhas ou massagens, dissipam uma resposta de relaxamento e bem-estar, através da liberação de mediadores químicos (ZOTELLI VLR, RANDO MMP, SOUSA MLR. 2010).

Este método que visa a prevenção e tratamento de dores, através do uso de inserção de agulhas em pontos anatômicos dentro da medicina chinesa, é denominado acupontos. Sua eficácia já vem sendo comprovada em vários estudos, tendo seus resultados comprovados cientificamente. No momento em que ocorre a aplicação da agulha de acupuntura, é desencadeado um processo de liberação química de reguladores na corrente sanguínea (endorfina, seretonia, $\beta$-endorfina), atuando assim no alívio de algias relacionadas aos pontos tratados (SANTOS J, RECCO P, MOTA G, HOLANDA AV, JUNIOR VES. 2017). 
Outro processo químico decorrente da inserção das agulhas do acupontos é a produção de histamina, prostaglandinas e íons potássio, que estimulam os quimiorreceptores, causando uma diminuição do limiar excitatório. Quando os acupontos são estimulados, ativam das fibras nervosas musculares, onde enviam impulsos para a medula espinal liberando agentes químicos, que resultam na inibição da dor. O bruxismo, tem como um dos sintomas a dor, tanto nos músculos mastigatórios como também presença de cefaleia nos pacientes bruxômanos, afetando assim sua qualidade de vida, trazendo consigo ansiedade, aumento da irritabilidade e limitações funcionais (SANTOS J, RECCO P, MOTA G, HOLANDA AV, JUNIOR VES. 2017).

O uso dessa técnica em pacientes com dor relacionadas ao bruxismo, tem se mostrado eficaz no aumento dos movimentos mandibulares, resultando do relaxamento dos músculos mastigatórios e analgesia duradora que o tratamento de acupuntura proporciona (SANTOS J, RECCO P, MOTA G, HOLANDA AV, JUNIOR VES. 2017).

Um estudo realizado, com o intuito de acompanhar um caso clínico de paciente com bruxismo do sono, com relato de dor principalmente do lado direito, foi sujeitado ao tratamento de acupuntura, em sessões semanais de 20 minutos. Demostrando uma redução da dor e melhoria na qualidade do sono. Levando a considerar que o uso da acupuntura como meio de terapia integrativa em pacientes com bruxismo, é uma ferramenta eficaz e de baixo custo (ZOTELLI VLR, RANDO MMP, SOUSA MLR. 2010).

A acupuntura, vê a patologia existente no organismo como resultado da influência da resposta do mesmo ao agente agressor, cujo sistema nervoso central é influenciado pelo corpo, de forma multifatorial. Mesmo a técnica de acupuntura sendo reconhecida como medicina alternativa, com comprovações cientificas de sua funcionalidade, e obtendo resultados consideráveis no tratamento do bruxismo, ainda existe uma carência de pesquisas e regulamentações sobre o uso da mesma em tratamentos odontológicos (ZOTELLI VLR, RANDO MMP, SOUSA MLR. 2010). 


\subsubsection{Farmacológicos}

Estudos apontam a existência de uma estrutura anatomia e funcional, específica por gerar movimentos oromandibulares involuntários, dentro sistema nervoso central. Onde apontam o envolvimento da neurotransmissão dopaminérgica e serotoninérgica na gênese assim como a estruturação do bruxismo. Existe duas havendo duas hipóteses aceitáveis para o surgimento desta disfunção: teoria hipodopaminérgica e teoria hiperdopaminérgica (RIVAS OS 2021).

A hiperdopaminérgicos podem ser desencadeada, pelo uso de ecstasy e cocaína, anfetaminas, no entanto, também podem se apresentar a pacientes com doença de Parkinson em uso de levodopa. Sendo nesses pacientes, mais predispostos para o surgimento de episódios de o ranger de dentes durante o sono. Assim como o uso continuo de drogas antidopaminérgicas, podem desenvolver hipersensibilidade dos receptores dopaminérgicos, promovendo assim, movimentos involuntários. ${ }^{18}$

$\mathrm{Na}$ hipótese hipodopaminérgica, é caraterizada pela utilização de antidepressivos inibidores seletivos da receptação de serotonina (ISRS). Sendo este, um dos maiores responsáveis pela inibição de serotonina, potencializando assim a neurotransmissão serotonérgica. Esse aumento por sua vez, exerce uma redução dopaminérgica nos sistemas nigroestriatal e mesocortical, ativando o córtex préfrontal, acarretando no ranger de dentes durante o sono (CORDEIRO PCD, REIS PLC. 2018).

O uso de fármacos na diminuição da atividade do bruxismo, tem sido muito difundido como meio terapêutico para essa disfunção, pois os mesmos atuam no mecanismo inicial do bruxismo, sendo sua indicação por um período curto e apenas em casos graves. A gama de medicamentos usados associados ao tratamento do bruxismo é muita vasta sendo os mais utilizados: Trazodona, medicamento utilizado para tratamento de insônia, apresentando efeito no bruxismo; Benzodiazepinicos, clonazepam apresentam com uma diminuição mais significativa com relação ao trazodona; Clonidina, sendo sua eficiência de até 60\% na redução do bruxismo (VASCONCELOS IGS, VENÂNCIO GN, SILVA FB. 2018). 
Outros fármacos são utilizados para o tratamento do bruxismo, mas sem o efeito esperado. Estudos feitos com grupos de pacientes, o qual uma parte realizou o uso de amitriptina $25 \mathrm{mg}$ e outro placebo, demonstrou por meio de um aparelho de eletromiografia portátil, que ambos os grupos não apresentaram mudança significativa da dor. Pesquisas similares foram realizados no uso de triptofano 5omg, L-dopa, bromocriptina de $1,25 \mathrm{mg}$ a $7,5 \mathrm{mg}$ e obtiveram os mesmos resultados. Estes mesmos estudos reforçaram a eficácia da clonidina e clonazepan do tratamento do bruxismo (FERREIRA JC. AFONSO. 2019).

Estudos realizados com io pacientes, em tratamento com placa oclusal, receberam clonazepam ou placebo e, posteriormente, foram submetidos a exames de polissonografia e psicometria, com diagnóstico sobre os pacientes que receberam I mg de clonazepam tiveram significativa melhora em episódios de bruxismo assim também, como melhor qualidade do sono (FERREIRA JC. AFONSO. 2019).

\subsubsection{Toxina Botulínica}

Dentro da Odontologia, a toxina botulínica é utilizada como terapia para a disfunção temporomandibular (DTM), no controle de cefaléia tensional, sorriso gengival, sorriso assimétrico, hipertrofia do masseter, dor orofacial, bruxismo entre outros. Demonstrando que sua utilização vem apresentando bons resultados e ampliando cada vez mais, técnicas dentro da odontologia (MARCIANO A. et al., 2014).

Estudos demonstraram que o bruxismo é acarretado devido a níveis elevados de atividade motora no músculo mandibular, sendo o bloqueio químico causado pela toxina botulínica eficaz no tratamento dessa disfunção (Figura 03). O efeito da toxina tem duração aproximada de 6 meses, porém falhas da dosagens e erros da técnica de aplicação podem diminuir esse tempo (MARCIANO A. et al., 2014).

O uso da Toxina Botulínica teve sua iniciação como agente terapêutico no ano de 1790 para o tratamento de estrabismo. Ao longo dos anos o uso da Toxina Botulínica tem se expandido em diversas áreas da saúde, desde tratamento estético a patológicos. Essa neurotoxina é originada pela bactéria Clostridium Botulinum e sua 
atuação funciona como um paralisador químico temporário dos músculos, causado pelo bloqueio da liberação de acetilcolina dos neurônicos motores (VASCONCELOS IGS, VENÂNCIO GN, SILVA FB. 2018).

Esse processo de paralisação muscular temporário pela toxina botulínica ocorre pelo bloqueio da ação da acetilcolina. A toxina, ao se ligar aos receptores na membrana pré-sináptica da terminação nervosa motora, os receptores desencadeiam uma reação de endocitose da neurotoxina. As moléculas são divididas em duas cadeias, sendo uma leve e outra pesada. A cadeia leve é transportada pela membrana da vesícula endocítica até o citosol. Esta divisão impende que as vesículas sinápticas se unem na parte interna da membrana celular, evitando assim a liberação de acetilcolina, que é responsável pela desenervação química. Sendo assim, o bloqueio permitirá um relaxamento e paralisação das fibras afetadas. Após um período de 4 a 6 meses, acontece a reestabilização da transmissão e função muscular (Esteves JLS. et al., 2017).

Figura 03 - Pontos de aplicação da Toxina Botulinica.

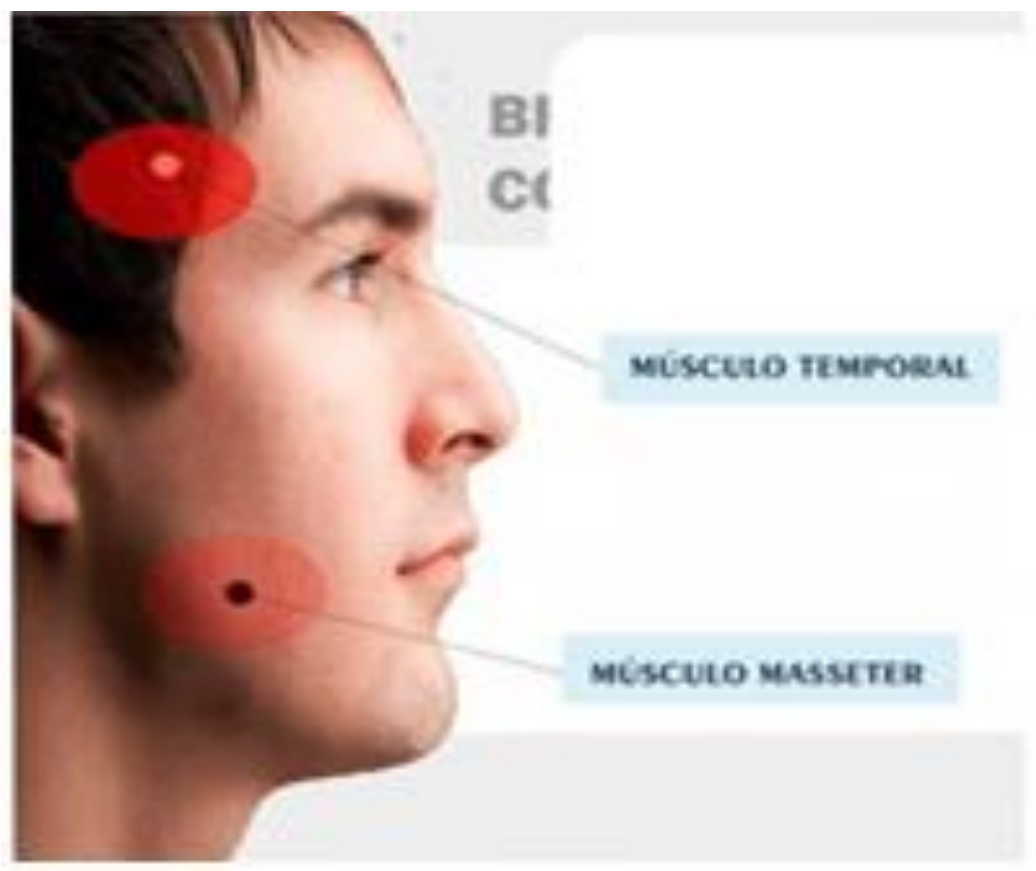

Fonte: Victor, Dentalis (202I) 
Um estudo realizado com 13 pacientes, que faziam uso de placas intraorais, por 2 meses, em que após o mesmo sem o uso da mesma, foram submetidos a 6oUI de Toxina Botulínica em ambos os lados do músculo masseter. O estudo mostrou que ambos os tratamentos apresentaram resultado significativo na redução da dor desses pacientes. Sendo assim, a Toxina Botulínica tendo o mesmo resultado que a placa, tornando-se uma sugestão eficaz no tratamento do bruxismo. O mesmo estudo avaliou estes pacientes com o exame de eletromiografia, observando uma diminuição notória na atividade do músculo masseter, ao mesmo tempo que o músculo temporal permanece inalterado. $\mathrm{O}$ uso da Toxina Botulínica, em casos graves de bruxismo, com reajuste de doses durante o tratamento, de acordo com a resposta ao tratamento, demostrou eficácia deste tratamento mesmo nos casos mais graves (LIMA LR, NECHITA HP. 2020).

Ocorreu também, novo estudo também voltado à utilização da toxina botulínica no tratamento de bruxismo severo e suas complicações. Neste estudo, foram avaliados 18 pacientes entre 20 a 50 anos de idade, onde todos os constituintes da pesquisa, relataram como principal sintoma o apertamento e ranger os dentes. A pesquisa teve como critérios de diagnósticos: dificuldade na fala, mastigação e deglutição, desgaste dentário, dor ou hipertrofia do músculo masseter e relato de familiares de ausculta de sons de ranger de dentes por parte dos participantes. $O$ tratamento foi composto por injeções de toxina botulínica no músculo masseter em dois a três pontos, seguindo uma dose de 25 a rooUI em cada lado do músculo, com um intervalo de cinco meses entre cada tratamento. Foi definida a duração do efeito, por vez da mensuração total das semanas em que os pacientes notaram melhora. Sendo que dos 18, 16 participantes relataram melhora no ranger dos dentes, mastigação e fala e apenas um paciente relatou sintoma adverso de disfagia. Demonstrando ao final do estudo que pacientes com bruxismo severo necessitam de injeções de toxina botulínica a cada 5 meses regularmente, sendo um tratamento eficaz, no entanto, custoso (LIMA LR, NECHITA HP. 2020).

Durante a utilização da toxina botulínica, algumas prevenções devem ser tomadas no que se diz respeito a paralisação dos músculos vizinhos, que não 
necessitam de tratamento, existindo uma possibilidade da mesma se espalhar, ocasionando alterações funcionais destes músculos. Os cuidados a serem tomados, estão relacionados a quantidade utilizada por aplicação, realizando aplicações individuais com o intuito de bloquear a contrações musculares e evitando a paralisação total do músculo. Os efeitos colaterais que são raros e breves, não causam graves complicações aos pacientes, são eles: náuseas, disfagia, hipersalivação, disfonia de palavras, hipotensão (LIMA LR, NECHITA HP. 2020).

Existem algumas contraindicações, como pacientes grávidas ou lactantes, alergia ao medicamento, região em processo infeccioso ou inflamatório. Em alguns casos, a contraindicação é relativa, como doenças neuromusculares, doenças autoimunes, coagulopatia descompensada, nessa circunstância deve ser feito uma avaliação minuciosa do custo benefício do tratamento. ${ }^{23}$

Ressalta-se ainda que a toxina botulínica, não atinge os Sistema Nervoso Central, não ultrapassando a barreira hemato-encefálica. Após 24 horas da aplicação da neurotoxina 60\% dela é expelida pela urina. Seu processo de metabolização é realizado através da protease e outros componentes, por metabolismo normal (MACHADO L, SOUSA T, SALLES M. 2020).

\subsubsection{Estimulação Elétrica Nervosa Transcutânea}

Dentro dos meios terapêuticos existentes para o tratamento do bruxismo, está a Estimulação Elétrica Nervosa Transcutânea (TENS), atuando como terapia que equilibra a oclusão, auxiliando assim na redução da dor e tensão muscular. O TENS (Figura 04) é realizado utilizado de eletrodos fixados nos músculos masseter e temporal, que estão ligados a um aparelho que transmite uma corrente elétrica pulsada de baixa voltagem. $O$ aparelho é ajustado conforme a sensibilidade do paciente para evitar contrações musculares indesejadas, sendo seu intuito promover analgesia ou hipoestesia na região tratada (LIMA LR, NECHITA HP. 2020). 
Figura 04 Aparelho de Estimulação Elétrica Nervosa Transcutânea.

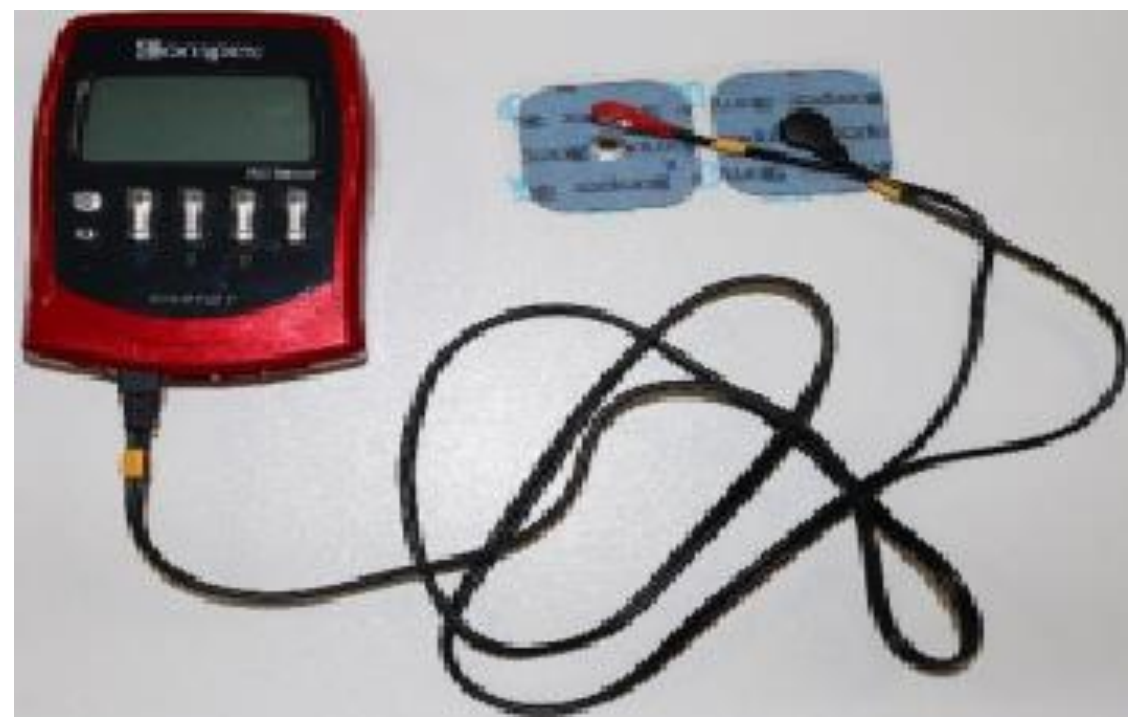

Fonte: NUNES, (2019)

Pacientes que utilizaram a TENS, obtiveram alívio da dor, simultaneamente com a diminuição dos movimentos musculares, segundo estudos. A associação do tratamento de TENS com placa miorrelaxante tem apresentado um efeito mais promissor, diminuindo significativamente a atividade eletromiográfica do músculo temporal durante o sono (LIMA LR, NECHITA HP. 2020).

Um estudo realizado com um grupo de bruxomonos, o qual foi classificado a sintomatologia dolorosa em: pico, média e atual, com o intuito de mensurar a dor dos participantes, demostrou que a terapia TENS teve efeito mais significativo na dor de maior intensidade, o que refere o seu benefício da Estimulação Elétrica Nervosa Transcutânea no controle da dor, sendo eficaz no tratamento do bruxismo. No entanto a associação desse tratamento com demais formas terapêuticas é fundamental, pois devido ao fato de ser uma parafunção relacionada a regulação central, o estado psicológico está fortemente relacionado aos episódios de bruxismo (TEIXEIRA SAF. 2013).

O TENS, é classificado por dois grupos; o de baixa frequência, menor de ro $\mathrm{Hz}$, e o de alta frequência, superior a $50 \mathrm{~Hz}$, sendo o usado no tratamento 
odontológico com utilização mista, com corrente de baixa frequência, para a ação de relaxamento muscular e alta frequência para evitar contrações musculares, obtendo assim uma analgesia muscular (GROSSMANN E. et al., 2012).

O mecanismo de atuação do TENS se baseia na estimulação dos nervos motores, promovendo contrações rítmicas, aumentando a circulação sanguínea na região, desta forma reduzindo o edema intersticial, diminuindo assim a dor e fadiga dos músculos mastigadores. Outra linha de estudo relacionada a teoria do portão da dor dentro da eletroanalgesia, o qual propõe a existência de um elo na medula espinal que regula a entrada nociceptiva através das fibras nervosas, sendo essa anulada através de estímulos táteis ou correntes elétricas (GROSSMANN E. et al., 2012).

\subsubsection{Laser de baixa potência}

A terapia com laser é utilizada em diversos ramos da saúde, atualmente seu uso dentro da Odontologia está sendo bastante difundido, como no tratamento a pacientes bruxononos, com a utilização do laser de baixa potência. O laser atua na regeneração tecidual e alívio da dor, sendo uma das principais vantagens, não ser invasivo. No entanto, ainda se precisa mais pesquisas para uma padronização do tratamento de forma mais eficaz (SANTOS J, RECCO P, MOTA G, HOLANDA AV, JUNIOR VES. 2017).

O laser de baixa intensidade, beneficia a flexibilidade da musculatura reduzindo assim a dor, um efeito não obtido em tratamentos convencionais como a placa oclusiva, tornando possível, uma associação terapêutica. No entanto, a mensuração do efeito do tratamento, se torna limitado, sendo a dor subjetiva, a sua descrição se torna variável para cada paciente. (TEIXEIRA SAF. 2013).

Um estudo realizado com grupos separados, o qual o primeiro realizou tratamento com laserterapia, o segundo grupo recebeu o mesmo tratamento associado ao uso de placa oclusiva (Tabela or), mostrou redução significativa da dor em ambos os grupos, no entanto, o resultado foi mais significativo no grupo com a terapia associada, assim como a ampliação dos movimentos da mandíbula (MACHADO L, SOUSA T, SALLES M. 2020). 
Tabela or - Frequência dos sinais e sintomas nos dois grupos Estudados. Ar = antes do tratamento; $\mathrm{A}_{2}$ = após o tratamento; $\mathrm{GP}$ = grupo placa, $\mathrm{GPL}=$ placa-laser.

\begin{tabular}{|c|c|c|c|c|}
\hline \multirow{2}{*}{$\begin{array}{l}\text { Sinais e } \\
\text { sintomas }\end{array}$} & \multicolumn{2}{|c|}{ AI } & \multicolumn{2}{|c|}{ A2 } \\
\hline & GP & GPL & GP & GPL \\
\hline Dor muscular & 15 & Io & 8 & 2 \\
\hline $\begin{array}{l}\text { Fadiga } \\
\text { muscular }\end{array}$ & 12 & 9 & 9 & 2 \\
\hline Dor na ATM & 15 & 7 & 7 & 2 \\
\hline Ruídos na & 15 & 8 & 8 & 4 \\
\hline ATM & & & & \\
\hline Dor de cabeça & 15 & 9 & 7 & 2 \\
\hline Otalgia & 9 & 5 & 4 & o \\
\hline Zumbido & 9 & 6 & 6 & 2 \\
\hline $\begin{array}{l}\text { Plenitude } \\
\text { aural }\end{array}$ & 12 & 8 & 6 & I \\
\hline Dificuldade & & & & \\
\hline Abrir a boca & Io & 8 & 5 & I \\
\hline Fechar a boca & 5 & 4 & 2 & $\mathrm{o}$ \\
\hline Mastigar & II & 7 & 5 & 3 \\
\hline Bocejar & 9 & 9 & 5 & 6 \\
\hline Engolir & 6 & 3 & 2 & o \\
\hline Falar & 7 & 3 & 4 & o \\
\hline
\end{tabular}

Fonte: MELCHIOR, (2017).

Segundo o estudo realizado, em que foram registrados os sintomas relacionados ao bruxismo, em dois grupos, foram comparados os dados antes do tratamento e depois de 5 semanas em uso de placa oclusiva e associação do mesmo com laserterapia, observando assim uma redução considerável no número de sintomas em ambos os grupos, se sobressaindo o que foi submetido a terapia 
associada. Portanto o resultado do uso de laser de baixa intensidade no tratamento do bruxismo é potencializado em uso conjunto com a placa de oclusão (MACHADO L, SOUSA T, SALLES M. 2020).

\section{DISCUSÃO}

Sendo o bruxismo uma desordem da musculatura da mastigação, tendo como principal sintoma o apertamento dental ou ranger dos dentes, os efeitos prejudiciais oriundos desse comportamento mostram-se como justificativa para condutas de controle. Para tal, existem alguns manejos mais difundidos dentro do meio odontológico como a placa oclusiva, que pode ser de material vinil ou acrílico rígido. No entanto o uso de placa oclusiva minimiza apenas os danos. Outras terapias têm mostrado um resultado muito satisfatório no manejo do bruxismo, como a acupuntura, que obteve resultados consideráveis no alívio da dor duradoura e relaxamento muscular, assim também como diminuição da irritabilidade da melhora do sono do paciente com bruxismo.

O TENS segue também a linha de tratamento buscando alívio da dor e diminuição da fadiga muscular, através de estímulos dos nervos motores, promovendo contrações rítmicas, aumentando a circulação sanguínea na região, desta forma reduzindo o edema intersticial. Outra forma de tratamento que demostrou eficiência é o laser de baixa intensidade, que beneficia a flexibilidade da musculatura reduzindo assim a dor, um efeito não obtido em tratamentos convencionais como a placa oclusiva, tornando possível, uma associação terapêutica. Mas devido a dor ser subjetiva, se dificulta a mensuração do efeito do tratamento, sendo variável para cada paciente.

A toxina botulínica vem apresentando bons resultados no manejo do bruxismo, com alívio da dor devido à paralisação temporária de algumas fibras do músculo masseter, reduzindo, desta maneira, a força muscular e em consequência, a geração de mediadores algogênicos; no entanto, seu tratamento se torna custoso e os resultados de curto período de tempo. 
Já o uso de medicamentos para outras patologias, como a doença de Parkinson, hipertensão arterial, ansiolíticos e antidepressivos, quando usados no manejo do bruxismo têm se apresentando vantajosos pelo seu resultado positivo no controle de episódios de bruxismo, sendo sua indicação por um período curto e apenas em casos graves, principalmente com o uso de Trazodona, benzodiazepínicos, Clonidina, amitriptina, Levodopa, bromocriptina.

\section{CONSIDERAÇÕES FINAIS}

O bruxismo como uma desordem multifatorial, decorrente de uma série de fatores etiológicos. Dispõem de diversos métodos terapêuticos, no entanto, existem alguns manejos mais difundidos dentro do meio odontológico como a placa oclusiva, acupuntura, TENS, o laser de baixa intensidade, toxina botulínica, onde vem apresentando bons resultados no manejo do bruxismo. A qual, através de uma avaliação minuciosa, o profissional dentista poderá optar pelo método mais adequado, para a necessidade do paciente.

O uso de fármacos no manejo do bruxismo do sono tem apresentado melhor resultado na redução dos episódios de bruxismo, consequentemente diminuindo a dor e melhorando o sono do paciente, proporcionando assim, melhor qualidade de vida. Portanto, o manejo farmacológico, assim como o manejo psicológico e as terapias locais de redução da dor causada pelo bruxismo, mostram-se como alternativas para o uso da placa oclusal, que antes era a única forma conhecida de manejo do bruxismo.

\section{REFERÊNCIAS}

AMERICAN Sleep Disorders Association. International classification of sleep disorder: diagnostic and coding manual, revised. 2nd ed. Westchester, 2005. p. I89192.

ATTANASIO R. An overwiew of bruxism and its management. Orofacial Pain and related Disorders. 1997; 4I(2): 229-24I. 
BLINI, CC. et al..Relação entre bruxismo e o grau de sintomatologia de disfunçãotemporomandibular. Rev. CEFAC [online]. 2010, vol.12, n.3, pp.427-433. Epub May 21, 2010. ISSN 1516-1846. Disponível em;.

CORDEIRO PCD, Reis PLC. Polimorfismo No Gene Mmpz Está Associado Com Ausência De Desordens Temporomandibulares Musculares. Programa De PósGraduação Em.

CHASE MH, Morales, FR. Control of motoeurons during sleep. (Ed.). Principles and practice of sleep medicine. Philadephia: WB Saunders, 2000. p. 155-168.

DORLAND, WA. Dorland's illustrated medical dictionary. 29th ed. Philadelphia: W.B. Saunders, 200o. E.FAULKNER, K. B. D. Bruxism: a review of the literature. Part I.Aust. Dent. J., Sydney, v. 35, no. 3, p. 266-276, June I99o.

ESTEVES JLS. et al. Uso Da Acupuntura No Tratamento De Bruxismo. Revista da Universidade Vale do Rio Verde, Três Corações, v. I5, n. I, p. 763-773, jan./jul. 2017. Disponível em:< http://periodicos.unincor.br/index.php/revistaunincor/article/view/4I40 > Acesso em: ro de mai.21.

FERREIRA JC. Afonso. Avaliação Do Biofeedback Eletromiográfico No Tratamento Do Bruxismo. - 2019. 88 f.; il. Universidade Federal De Pernambuco Disponível em< https://repositorio.ufpe.br/bitstream/123456789/36059/r/DISSERTA\%C3\%87\%C3\% $83 \mathrm{O} \% 2$ oJessica\%20Caroline\%20Afonso\%2oFerreira.pdf > Acesso em I2 de mai.21.

GAIDA, PS. Bruxismo - Um Desafio para a Odontologia. 2004. $5 \mathrm{f}$.

GROSSMANN E. et al. O Uso Da Estimulação Elétrica Nervosa Transcutânea Na Disfunção Temporomandibular. Artigos de Revisão - Rev. dor 13 (3) - Set 2012. Disponível em:

LAVIGNE GJ. Bruxism physiology and pathology: an overview for clinicians Surgery Department, Pain, Sleep and Trauma Unit, Université de Montréal, Hôpital du Sacré-Coeur de Montréal, C.P. 6128, succ Centre ville, Montréal, $\mathrm{H}_{3} \mathrm{C}$ 3J 7, Canada. First published: o9 Junho 2008. Disponível em: 
LOPES, NM de A. Prevalência De Bruxismo Do Sono Em Pacientes Edêntulos Totais E A Concordância Entre Diferentes Métodos De Diagnóstico. Universidade Federal De Santa Catarina Centro De Ciências Da Saúde Departamento De Odontologia Curso De Graduação Em Odontologia. Florianópolis2or8.Disponívelem:< https://repositorio.ufsc.br/handle/123456789/187282

LOBBEZOO F, Lavigne GJ. Do bruxism and Temporomandibular Disorders Have Cause-andEffect Relationship? Journal of Orofacial Pain. 1997; II: 15-23 21 Lima LR, Nechita HP. Toxina botulínica na odontologia: Tratamento De Bruxismo Revisão De Literatura. Universidade São Francisco Odontologia. Bragança Paulista 2020.

.LOAIZA GAL. Diagnóstico E Tratamento Do Bruxismo Em Pacientes Jovens Revisão Bibliográfica. Mestrado Integrado em Medicina Dentária Instituto Universitário de Ciências da Saúde.2019. Disponível em:< https://repositorio.cespu.pt/bitstream/handle/20.500.11816/3159/MIMD_RE_26668_ Gustavo Loaiza.pdf? sequence $=\mathrm{I} \&$ is Allowed $=\mathrm{y}>$

LEITE LGM. Placa para bruxismo macia ou rígida: acerte no material ideal [dados digitais]. Disponível em:

MELCHIOR MO. et al. Laserterapia De Baixa Intensidade Associada Ao Uso De Placa Oclusal No Tratamento De Disfunção Temporomandibular: Estudo Clínico Controlado. Rev Dor. São Paulo, 2017 jan-mar;I8(I):12-7

MACHADO L, Sousa T, Salles M. Toxina Botulínica e seu uso No Tratamento do Bruxismo. Original Article. J Business Techn. 2020; ISSN 2526-428I I6(I): I08-I2I MARCIANO A. et al. Toxina Botulínica E Sua Aplicação Na Odontologia. Revista de Iniciação Científica da Universidade Vale do Rio Verde, Três Corações, v. 4, n. I, 2014, p. $\quad 65-75 . \quad$ Disponível em:< http://periodicos.unincor.br/index.php/iniciacaocientifica/article/view/I554/I218> Acesso em: ro de mai.21.

MACHADO E. et al. Bruxismo do sono: possibilidades terapêticas baseadas em evidências. Dental Press J Orthod 20II Mar-Apr;16(2):58-64. Disponível em:< 
https://www.scielo.br/scielo.php?pid=S2176-

94512011000200008\&script=sci_abstract\&tlng=pt > Acesso em o9 de mai.21.

MANFREDINI D. et al. Epidemiology of bruxism in adults: a systematic review of the literature. J Orofac Pain. 2013b;27:99-110

Nascimento AM. et al. Associação do bruxismo ao estresse emocional: estudo transversal. RBO. 2009; 66 (2): 160-4.

NUNES RFS. Estimulação Elétrica Nervosa Transcutânea (TENS) no controlo da Dor Miofascial associada ao Bruxismo. Universidade de Lisboa Faculdade de Medicina Dentária. Dissertação Mestrado Integrado em Medicina Dentária.2org. Disponível em:< https://repositorio.ul.pt/bitstream/I0451/41298/I/ulfmd_o8816_tm_Ricardo_Nunes.p df >

RIOS LT, Aguiar VNP, Machado FC, Rocha CT, Neves BG. Bruxismo infantile sua associação com fatores psicológicos - revisão sistemática da literatura. Rev. Odontol. Univ. Cid. São Paulo 2018 jan-mar; 30(I): 64-76

RIVAS PS. O Uso Dos Dispositivos Oclusais No Bruxismo Do Sono. Dissertação conducente ao Grau de Mestre em Medicina Dentária. Instituto Universitário de Ciências de Saúde. Gandra, I6 de jun. 2I. Disponível em:< https://repositorio.cespu.pt/handle/20.500.11816/3566> Acesso em io de mai.2I.

Santos J, Recco P, Mota G, Holanda AV, Junior VES. Tratamento da dor orofacial através da acupuntura em pacientes com bruxismo: um estudo de revisão. RFO, Passo Fundo, v. 22, n. I, p. 96-Ioo, jan/abr. 2017.

TEIXEIRA SAF. A utilização de Toxina botulínica para bruxismo: Revisão de Literatura. Rev. bras. odontol., Rio de Janeiro, v. 70, n. 2, p. 202-4, jul./dez. 2013. Disponívelem:<

http://revodonto.bvsalud.org/scielo.php?script=sci_arttext\&pid=Soo3472722013000200021> Acesso em: io de mai.21.

VICTOR. Toxina botulínica: surge como possibilidade no tratamento do bruxismo. Dentalis sempre a mão [Dados digitais] Victor • abr 04, 2018. Disponível em:< 
https://www.dentalis.com.br/blog/toxina-botulinica-surge-como-possibilidade-notratamentodo-bruxismo>.

ZOTELLI VLR, Rando MMP, Sousa MLR. Uso da acupuntura no manejo da dor em pacientes com alterações na articulação temporomandibular (ATM). Revista de Odontologia da Universidade Cidade de São Paulo 2010; 22(2): 185-8, mai-ago. 